\title{
Incidencia de los conocimientos biológicos de la homosexualidad y transexualidad sobre la homofobia y transfobia en estudiantes de educación física de Chile \\ Incidence of the biological knowledge of homosexuality and transsexuality on homophobia and transphobia in physical education students in Chile
}

Fernando Maureira Cid*, Natalia Escobar Ruiz**, Elizabeth Flores Ferro***, Marcelo Hadweh Briceño****, Patricia González Flores*****, Franklin Castillo-Retamal******, Sebastián Peña-Troncoso*******, Jacqueline Páez Herrera********

*Universidad Metropolitana de Ciencias de la Educación (Chile), **Universidad Bernardo O’Higgins/Universidad SEK (Chile), ***Universidad Bernardo O'Higgins, ****Universidad SEK (Chile), *****Universidad Católica Silva Henríquez (Chile); ******Universidad Católica del Maule (Chile), $* * * * * * *$ Universidad Austral de Chile (Chile), $* * * * * * * *$ Pontificia Universidad Católica deValparaíso (Chile)

Resumen. Introducción: Diversos estudios relacionan variables como la edad, sexo, nivel socioeconómico, religión, etc. con los niveles de homofobia y transfobia. El objetivo de la presente investigación fue determinar la incidencia de algunas variables sociodemográficas y de los conocimientos biológicos sobre la homosexualidad y transexualidad sobre los niveles de homofobia y transfobia en estudiantes de Educación Física de Chile. Método: se aplicó una encuesta sociodemográfica, la escala de homofobia EHF-10, la escala de actitudes negativas hacia personas trans y el cuestionario de conocimientos biológicos de la homosexualidad y transexualidad a 463 estudiantes de Pedagogía en Educación Física de seis universidades de Chile. Resultados: los niveles de homofobia y transfobia son bajos en la muestra, siendo la convivencia con amigos/as o conocidos/as homosexuales, bisexuales y trans, y las creencias religiosas los factores que más inciden en dichas actitudes negativas. Se observó una relación negativa y baja entre conocimientos biológicos de homosexualidad y transexualidad y los niveles de homofobia y transfobia. Conclusión: la muestra presenta bajos niveles de homofobia y transfobia. También se observan bajos valores de conocimientos biológicos de la homosexualidad y transexualidad, con una relación baja y negativa entre dichos conocimientos y las actitudes negativas hacia personas homosexuales y trans.

Palabras claves: homosexualidad, transexualidad, biología, educación física, estudiantes.

\begin{abstract}
Introduction:Various studies relate variables such as age, sex, socioeconomic level, religion, etc. with levels of homophobia and transphobia. The aim of this research is to determine the incidence of some sociodemographic variables and biological knowledge about homosexuality and transsexuality on the levels of homophobia and transphobia in physical education students in Chile. Method: a sociodemographic survey was applied, the scale of homophobia EHF-10, the scale of negative attitudes towards trans people and the questionnaire of biological knowledge of homosexuality and transsexuality to 463 students of Pedagogy in Physical Education from six universities in Chile. Results: The levels of homophobia and transphobia are low in the sample, being the coexistence with friends/ or known/ homosexual, bisexual and trans, and the religious beliefs the factors that most affect those negative attitudes. A negative and low relationship was observed between biological knowledge of homosexuality and transsexuality and levels of homophobia and transphobia. Conclusion:The sample has low levels of homophobia and transphobia.There are also low biological knowledge values of homosexuality and transsexuality, with a low and negative relationship between such knowledge and negative attitudes towards homosexuals and transgender people.
\end{abstract}

Keywords: homosexuality, transsexuality, biology, physical education, students.

\section{Introducción}

La orientación sexual puede ser definida como una atracción afectiva, romántica y sensual hacia alguien de sexo opuesto, el mismo sexo, ambos o ninguno (American Psychological Association, 2011). En relación con esto, la homofobia se define como el sentimiento de rechazo, temor o repugnancia hacia una persona homosexual (Campo et al. 2013). Por su parte, la identidad de género corresponde a la autopercepción subjetiva que cada individuo tiene sobre su género (Maureira, 2020), la cual puede coincidir con el sexo

Fecha recepción: 16-07-20. Fecha de aceptación: 27-06-20 Fernando Maureira

maureirafernando@yahoo.es asignado al nacer (cisgénero) o puede ser diferente (transgénero). En relación con lo anterior, la transfobia corresponde a la discriminación y rechazo hacia las personas denominadas como transgénero o transexual (Barrientos et al. 2019).

Diversos estudios relacionan variables como la edad, sexo, nivel socioeconómico, religión, etc. con los niveles de homofobia y transfobia. Oltra et al. (2017) estudiaron la relación entre los niveles de homofobia y variables de personalidad, edad, sexo y clase social en 521 españoles con edades entre 16 y 68 años. Los resultados arrojaron que existe relación entre la homofobia y los factores de personalidad (Big five) correspondientes a Neuroticismo $(r=.117)$, Extraversión $(r=-.159)$, Apertura $(r=-.398)$, Amabilidad $(r=-.280)$ y Responsabili- 
$\operatorname{dad}(r=-.094)$. Se encontraron diferencias entre los niveles de homofobia según sexo $(p=.001$ con mayor puntaje en hombres) y clases sociales de la muestra $(p=.03$ con mayor puntaje en la clase baja y medioalta), pero no en la variable edad.

Otra investigación llevada a cabo por NietoGutiérrez et al. (2019) en 883 estudiantes de medicina de once universidades peruanas, muestra que menores niveles de homofobia se relacionan con ser mujer $(p=.005)$, estudiar en una universidad de Lima $(p=.001)$, profesar la religión católica $(p=.001)$, conocer a un paciente homosexual $(p=.003)$ y haber atendido a un paciente homosexual $(p=.036)$. Sin embargo, la variable ser machista aumentó la homofobia $(p=.007)$. Los autores concluyen que los varones tienden a ser más homofóbicos, lo que podría perjudicar la formación en pregrado y la relación médico-paciente a futuro. Hatibovic et al. (2017) compararon los niveles de homofobia y xenofobia según sexo, religión y orientación política en una muestra de 509 estudiantes universitarios de Valparaíso de Chile. Los resultados evidencian que las mujeres son menos homofóbicas que los hombres $(p<.01)$, que los sujetos que se declararon creyentes poseen niveles más altos de homofobia $(p<.001)$ y que los participantes que se declaran de izquierda son menos homofóbicos $(p<.001)$.

Kwak et al. (2019) estudiaron los niveles de homofobia e identificaron los factores que inciden en ella, en una muestra de 265 estudiantes coreanos de la carrera de enfermería. Los resultados arrojaron que el 92.9\% de los estudiantes fueron clasificados como homofóbicos y un 42.3\% como altamente homofóbicos. Además, encontraron que los participantes hombres, religiosos, con problemas de baja autoestima sin una persona cercana que perteneciera a un grupo minoritario sexual tenían mayores probabilidades de ser homofóbicos. Los autores concluyen que los elevados índices de homofobia de estudiantes de enfermería podrían afectar la gestión de salud y que son necesarios programas educativos para reducir los niveles de rechazo a homosexuales en dicha población. Colonnello et al. (2020) analizaron la relación entre variables sociodemográficas y actitudes hacia la homosexualidad en 658 estudiantes de medicina de universidades italianas. Los resultados mostraron una asociación negativa entre la capacidad para atender pacientes homosexuales y los niveles de homofobia $(r=-.487 ; p<.05)$. Las variables sexo masculino $(b=.347 ; p=.03)$, ser soltero $(b=.417 ; p<.02)$, orientación política moderada $(b=1.2$; $p=.04)$, postura política conservadora $(b=1.8 ; p=.02)$ y los sujetos declarados como religiosos $(b=.02 ; p=.001)$ tienen los niveles más alto en la predicción de la homofobia. Al mismo tiempo, el grupo con los niveles más altos de homofobia presentan el porcentaje más deficiente acerca del conocimiento de la homosexualidad. Los autores concluyen que la actitud homofóbica se correlaciona con menor conocimiento acerca de la homosexualidad, lo que podría poner en riesgo el profesionalismo del futuro médico.

Un estudio de Georgius et al. (2018) acerca de la actitud sobre la homosexualidad femenina y masculina en 552 estudiantes de educación física de la Universidad Nacional de Atenas en Grecia mostraron que participar en deportes no es un elemento que influya en las actitudes hacia la homosexualidad en los sujetos. Los factores como el género, religiosidad, inclinación o postura política y la socialización con una persona no heterosexual predicen las actitudes hacia los homosexuales. Los autores concluyen en la necesidad de estrategias antihomofóbicas e integrar una perspectiva de género que considere los prejuicios inconscientes, las políticas de diversidad y el seguimiento de estructuras organizacionales e institucionales para revertir las actitudes y acabar con la discriminación sistemática.

En relación con la transfobia, Álvarez-Conde y Rodríguez-Castro (2017) evaluaron a 149 estudiantes universitarios de educación primaria de España (32.2\% hombres y $67.8 \%$ mujeres) con un instrumento sobre el conocimiento de la sexualidad, un inventario de sexualidad ambivalente, una escala de transfobia, una escala de opinión sobre la sexualidad y un cuestionario sobre si tenían en su plan de estudio alguna cátedra de sexualidad y si creen que es necesario en su proceso formativo. Los principales resultados muestran que el 80\% tienen un alto nivel de desconocimiento sexual y un $90 \%$ no tiene conocimientos generales sobre identidad corporal y de género. Los hombres presentan mayor nivel de sexismo, tanto benevolente como hostil. Misma situación ocurre con los sujetos que se describen como más religiosos, siendo los que presentan mayor sexismo y transfobia. Por su parte, también encontraron que el hecho de haber tenido una relación de pareja anteriormente podría implicar actitudes más positivas hacia la sexualidad. Finalmente, las personas que consideran necesaria una formación sexual durante su formación académica poseen menor sexismo benevolente, hostil y transfobia.

Por su parte, García-Barba et al. (2018) estudiaron las actitudes hacia la transexualidad en 179 jóvenes españoles con una media de edad de 22,8 $\pm 2,2$ (103 fue- 
ron mujeres y 76 hombres). Los principales resultados muestran porcentajes altos de actitudes negativas hacia la transexualidad entre los sujetos evaluados, siendo los hombres los que poseen mayores puntuaciones. Por otra parte, el hecho de conocer o no a una persona trans influye significativamente, pues los jóvenes que no conocen a ninguna persona transexual presentan mayores niveles de actitudes negativas hacia dichas personas. Acker (2017) evalúo los niveles de transfobia en 600 estudiantes de trabajo social, terapia ocupacional y enfermería de una universidad pública de la ciudad de New York con el objetivo de analizar los efectos de los contenidos transgénero en la educación, religión, contacto con personas trans y otras variables sociodemográficas. Los principales resultados fueron que un 75\% de los alumnos señalan como deficiente el contenido transgénero en su formación y casi la mitad de la muestra mostró niveles moderados a altos de transfobia. Las principales conclusiones del estudio, es que se sugiere aumentar el contenido transgénero en libros de texto, conferencias y/o reflexiones en clases, así como generar instancias de estos profesionales para poder trabajar con dicha población.

García-Acosta et al. (2020) aplicaron la escala de actitudes negativas hacia personas trans a un total de 602 estudiantes y profesionales de la salud de Tenerife. Los principales resultados muestran que tanto hombres como mujeres son menos transfóbicos si las identidades de las personas trans coinciden con la de ellos. Los autores concluyeron que la muestra estudiada posee bajos niveles de transfobia y que las principales barreras percibidas por las personas trans pueden estar dadas por falta de formación y conocimiento más que de prejuicios.

Con la finalidad de profundizar en el tema de la variables que inciden en la homofobia y transfobia, es que surge el objetivo de la presente investigación: determinar la incidencia de variables sociodemográficas como la edad, sexo al nacer, creencias religiosas, amistades o conocidos homosexuales y transgénero, educación sexual recibida, nivel de ingreso de los participantes y conocimientos biológicos sobre la homosexualidad y transexualidad sobre los niveles de homofobia y transfobia en estudiantes de Educación Física de Chile.

\section{Método}

\section{Muestra}

De tipo no probabilística intencionada (Maureira \& Flores, 2018). Estuvo constituida por 463 estudiantes de
Pedagogía en Educación Física de seis universidades de Chile, cinco de ellas privadas y una pública. Tres de las universidades se ubican en la Región Metropolitana, una en la región de Valparaíso, una en la región del Maule y otra en la región de Los Ríos. La edad mínima de la muestra fue de 18 y la máxima de 37 años, con una media de 21.4 22.8. Del total, 208 fueron hombres (44.9\%) y 255 fueron mujeres (55.1\%). 109 estudiantes cursaban primer año de la carrera (23.5\%), 103 segundo año (22.2\%), 112 tercer año (24.2\%), 74 cuarto año $(16.0 \%)$ y 65 quinto año (14.0\%). En cuanto a la orientación sexual, 384 estudiantes declararon ser heterosexuales (82.9\%), 23 dijeron ser homosexuales (5\%) y 56 se identificaron como bisexuales (12.1\%).

\section{Instrumentos}

Se utilizó una encuesta sociodemográfica que incluye información sobre la edad, el sexo al nacer, año que cursa en pedagogía en Educación Física, si están solteros/as o en una relación, creencias religiosas, si poseían conocidos o amigos/as homosexuales, bisexuales y transgénero, si recibieron educación sexual en colegios o universidades, si en caso de recibir educación sexual se trataron los temas de homosexualidad e identidad de género y el nivel de ingreso económico de los participantes.

Se aplicó la escala de homofobia EHF-10 (Moral \& Ortega, 2008) validada en estudiantes de Educación Física de Chile por Maureira et al. (2022). La escala consta de 10 ítems. El contenido del instrumento incluye la aceptación/exclusión de personas homosexuales, de la homosexualidad como orientación sexual y las manifestaciones de la homosexualidad en público. El análisis psicométrico entregó la existencia de un factor que explica el $58.1 \%$ de la varianza total, con un alfa de Cronbach de .761. La escala se contesta con un formato Likert de 1 (totalmente en desacuerdo) hasta 5 (totalmente de acuerdo).

Se utilizó la Escala de Actitudes Negativas hacia personas Trans (EANT) (Páez et al., 2015) validada en estudiantes de Educación Física de Chile por Maureira et al. (2022). La escala está constituida por 9 ítems. El contenido del instrumento incluye actitudes hacia personas trans y transfobia. El análisis psicométrico entregó la existencia de un factor que explica el 59.0\% de la varianza total, con un alfa de Cronbach de .901. La escala se contesta con un formato tipo Likert de 1 (muy en desacuerdo) hasta 5 (muy de acuerdo).

Finalmente, se aplicó el cuestionario de Conocimientos Biológicos de la Homosexualidad y Transexualidad 
(CBTH) construido y validado en estudiantes de Educación Física de Chile por Maureira (2021). El instrumento está constituido por 18 ítems (13 sobre homosexualidad y cinco sobre transexualidad) que corresponden a afirmaciones sobre aspectos cerebrales, hormonales y genéticos que subyacen a la homosexualidad y la transexualidad. El cuestionario consta de seis dimensiones: genes y hormonas en la homosexualidad; cerebro, homosexualidad y transexualidad; diferencias cerebrales homosexuales y transexuales; hormonas y homosexualidad; orientación sexual y transexualidad; homosexualidad humana y animal. Estos factores explican el $58.4 \%$ de la varianza total, con un valor de alfa de Cronbach de .763. El evaluado debe contestar «Correcto», «Incorrecto» o «No sé» frente a cada ítem.

\section{Procedimiento}

Se aplicaron la escala de homofobia EHF-10, la Escala de Actitudes Negativas hacia personas Trans y el cuestionario de Conocimientos Biológicos de la Homosexualidad y Transexualidad. Esto se realizó en forma online. Durante el presente estudio se respetaron los principios éticos para las investigaciones médicas con seres humanos de la Declaración de Helsinki (World Medical Asociation, 2013). Para participar en la presente investigación todos los estudiantes firmaron un consentimiento informado.

\section{Análisis de datos}

Para la presente investigación se utilizó el programa estadístico IBM SPSS versión 25.0 para Windows. Se aplicó estadística descriptiva (con medias, desviaciones estándar y tablas de frecuencia). También se realizaron pruebas de normalidad con la prueba de Kolmogorov-Smirnov (KS) que entregó una distribución normal de las variables $(p>.05)$, por lo cual se procedió a utilizar estadística paramétrica como pruebas $\mathrm{t}$ para muestras independientes comparando los resultados de los instrumentos entre mujeres y hombres, entre estudiantes que tienen y no tienen algún amigo/a o conocido/a homosexual, bisexual y transexual, entre estudiantes que recibieron y no recibieron educación sexual en el colegio o universidad, y entre aquellos que recibieron y los que no recibieron información sobre la homosexualidad e identidad de género. También se aplicaron pruebas ANOVA comparando los resultados de los instrumentos entre cursos, estado civil, creencias religiosas, personas con que vive, nivel socioeconómico y orientación sexual. Finalmente, se utilizaron correlaciones de Pearson para determinar las relaciones entre los resultados de los instrumentos utilizados y pruebas de regresiones lineales jerárquicas para determinar la incidencia de las variables sociodemográficas sobre la homofobia y transfobia. Se consideró significativo valores $p<.05$. Para la descripción de las puntuaciones de cada ítem se utilizaron porcentajes y se utilizó pruebas de $\mathrm{x}^{2}$ para comparar los puntajes obtenidos entre hombres y mujeres. Se consideró significativo valores $p<.05$.

\section{Resultados}

En la tabla 1 se observan las medias de respuestas a cada ítem de la escala de homofobia EHF-10. Es posible notar que el ítem 1. La sola idea de sentirme atraído/a físicamente por personas de mi propio sexo me pone nervioso / a posee el puntaje más alto $(2.19 \pm 1.2)$. Por el contrario, el ítem 3. Me avergonzaría tener un hermano homosexual o hermana lesbiana obtuvo el puntaje más bajo (1.17士.5). Al comparar los resultados entre hombres y mujeres se observa que en tres ítems existen diferencias significativas revelando una mayor homofobia en los hombres. El ítem 1. La sola idea de sentirme atraído / a físicamente por personas de mi propio sexo me pone nervioso / a es la que presenta mayor diferencia con un puntaje más alto en los hombres.

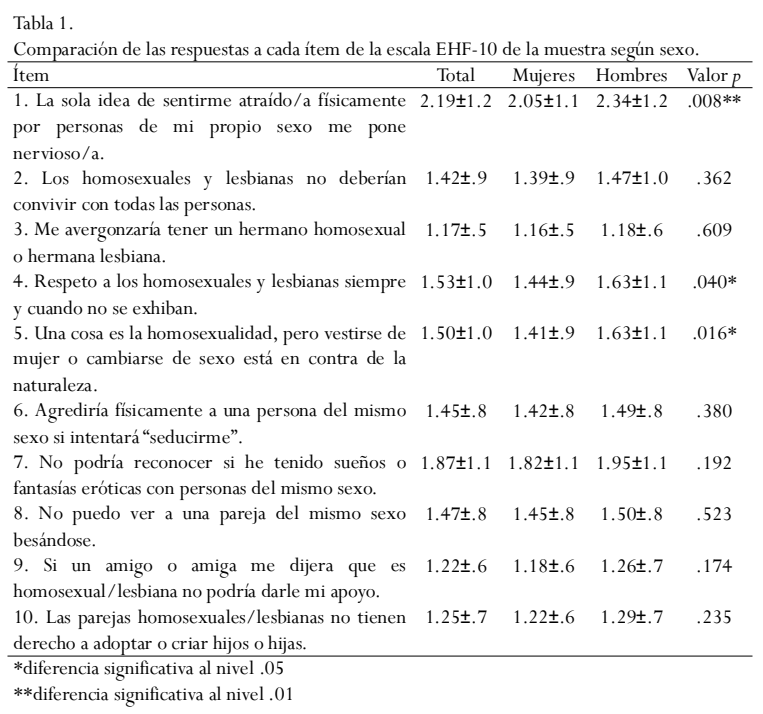

Al comparar las medias del puntaje total de la escala EHF-10 según el curso en que se encontraban los estudiantes, no se observaron diferencias significativas $\left(1^{\circ}\right.$ $\mathrm{año}=1.43 \pm .4 ; 2^{\circ}$ año $=1.57 \pm .5 ; 3^{\circ}$ año $=1.49 \pm .5 ; 4^{\circ}$ año $=1.47 \pm .5 ; 5^{\circ}$ año $\left.=1.47 \pm .5 ; p=.285\right)$. Misma situación ocurre al comparar entre los diversos niveles socioeconómicos de la muestra $(p=.790)$ y al comparar entre estudiantes que se describen como solteros/as y aquellos/as que se encuentran en una relación $(p=.126)$.

El grupo de estudiantes que poseen amigos/as o co- 
nocidos/as homosexuales o bisexuales obtuvieron un puntaje más bajo que aquellos que reportaron no tener$\operatorname{los}\left(\mathrm{Si}=1.45 \pm .5 ; \mathrm{No}_{1}=1.84 \pm .6 ; p=.000\right)$. La misma situación ocurre al comparar entre estudiantes que poseen amigos/as o conocidos/as transgéneros y los que no $\left(\mathrm{Si}=1.33 \pm .4 ; \mathrm{No}_{1}=1.57 \pm .5 ; p=.000\right)$.

Los estudiantes que recibieron educación sexual en su colegio o universidad obtuvieron puntajes más altos en homofobia que aquellos que reportaron no recibir dicha educación $\left(\mathrm{Si}=1.52 \pm .5 ; \mathrm{No}_{\mathrm{O}}=1.42 \pm .5 ; p=.038\right)$. Sin embargo, al comparar entre estudiantes que recibieron información sobre la homosexualidad e identidad de género en sus clases de educación sexual en su colegio o universidad y los que no, los análisis no revelan diferencias significativas $(\mathrm{Si}=1.51 \pm .5 ; \mathrm{No}=1.48 \pm$. 5 ; $p=.578)$.

Finalmente, al comparar las medias del puntaje de la escala EHF-10 de los estudiantes según su creencia religiosa el grupo que respondió ser ateo/a posee puntajes más bajos que el resto y el grupo evangélico logra las mayores puntuaciones en homofobia (Católi$\mathrm{co} / \mathrm{a}=1.54 \pm .5$; Evangélico $/ \mathrm{a}=1.84 \pm .6$; Ateo /

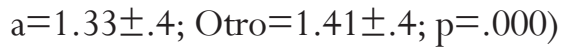

En la tabla 2 se observan las medias de respuestas a cada ítem de la Escala de Actitudes Negativas hacia personas Trans (EANT). Es posible notar que el ítem 8. Las personas trans son más propensas que el resto de la sociedad a contraer una enfermedad sexual posee el puntaje más alto (1.78土1.0). Por el contrario, el ítem 2. Las personas trans no deberían poder enseñar en las escuelas obtuvo el puntaje más bajo (1.28土.6). Al comparar los resultados entre hombres y mujeres se observa que en dos ítems existen diferencias significativas revelando una mayor transfobia en los hombres. El ítem 4. Las personas travestis son una amenaza para la familia y la sociedad es la que presenta mayor diferencia con un puntaje más alto en los hombres.

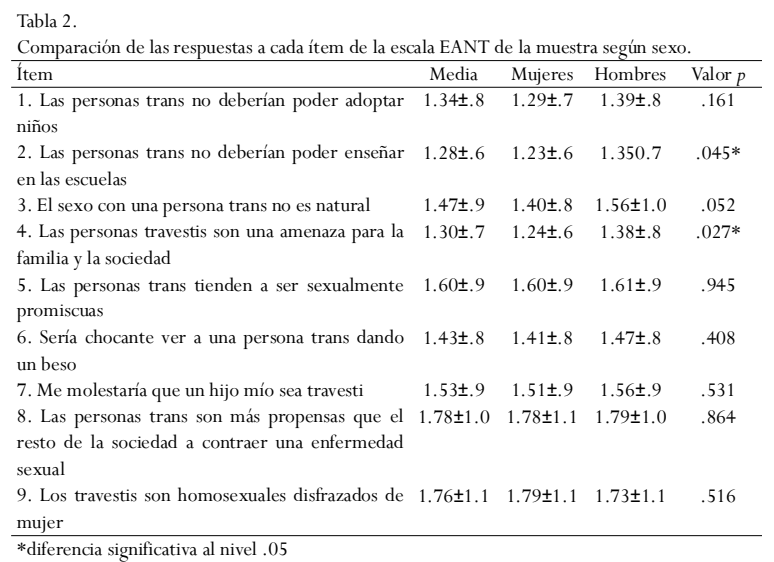

Al comparar las medias del puntaje total de la escala EANT según el curso en que se encontraban los estudiantes, no se observaron diferencias significativas $\left(1^{\circ}\right.$ año $=1.40 \pm .6 ; 2^{\circ}$ año $=1.53 \pm .6 ; 3^{\circ}$ año $=1.43 \pm .7 ; 4^{\circ}$ año $=1.55 \pm .7 ; 5^{\circ}$ año $\left.=1.51 \pm .6 ; p=.428\right)$. Misma situación ocurre al comparar entre los diversos niveles socioeconómicos de la muestra $(p=.578)$. Los estudiantes que se describen como solteros/as presentan puntajes más altos que aquellos/as que se encuentran en una relación (Solteros $=1.53 \pm .6$; En una relación $=1.39 \pm .6$; $p=.020)$.

El grupo de estudiantes que poseen amigos/as o conocidos/as homosexuales o bisexuales obtuvieron un puntaje más bajo que aquellos que reportaron no tener$\operatorname{los}\left(\mathrm{Si}=1.44 \pm .6 ; \mathrm{No}_{0}=1.87 \pm .7 ; p=.000\right)$. La misma situación ocurre al comparar entre estudiantes que poseen amigos/as o conocidos/as transgéneros y los que no $(\mathrm{Si}=1.29 \pm .5 ; \mathrm{No}=1.58 \pm .7 ; p=.000)$.

Los estudiantes que recibieron educación sexual en su colegio o universidad obtuvieron puntajes similares a que aquellos que no recibieron dicha educación $\left(\mathrm{Si}=1.51 \pm .6 ; \mathrm{No}_{0}=1.42 \pm .6 ; p=.136\right)$. Misma situación ocurre al comparar entre estudiantes que recibieron información sobre la homosexualidad e identidad de género en sus clases de educación sexual en su colegio o universidad y los que no $(\mathrm{Si}=1.50 \pm .6 ; \mathrm{No}=1.47 \pm .6$; $p=.652$ ).

Finalmente, al comparar las medias del puntaje de la escala EANT de los estudiantes según su creencia religiosa el grupo que respondió ser ateo/a posee puntajes más bajos que el resto y el grupo evangélico logra las mayores puntuaciones (Católico/a $=1.54 \pm .6$; Evangélico/a $=1.96 \pm .8 ; \quad$ Ateo/ $\mathrm{a}=1.28 \pm .4$; Otro $=1.39 \pm .6 ; p=0,000)$.

En la tabla 3 se observan los porcentajes de respuestas a cada ítem del cuestionario de Conocimientos Biológicas de la Homosexualidad y Transexualidad (CBHT). Es posible notar que el ítem 7. Existe un "gen gay" que determina la homosexualidad y el lesbianismo posee el mayor porcentaje de respuestas correctas (67.5\%). Por el contrario, el ítem 13. Cuando una mujer es lesbiana existen más probabilidad que su hermana también sea lesbiana obtuvo el porcentaje más bajo de respuestas correctas (1.3\%). El ítem 9. Existen más hombres homosexuales que mujeres lesbianas posee el porcentaje más alto de respuestas No sé con un $82.3 \%$.

El porcentaje total de respuestas correctas del cuestionario CBHT obtuvo un mínimo de $0 \%$, un máximo de $55.6 \%$ y una media de $19.2 \%$. No presenta diferencias según sexo de la muestra, curso de la carrera, entre 
Tabla 3.

Porcentaje de las respuestas a cada ítem del cuestionario CBHT del total de la muestra.

\begin{tabular}{|c|c|c|c|c|}
\hline Item & Respuesta & Correcto & Incorrecto & No sé \\
\hline $\begin{array}{l}\text { 1. Ninguna otra especie animal presenta } \\
\text { conductas homosexuales o lésbicas. }\end{array}$ & Incorrecta & $\begin{array}{c}15 \\
(3.2 \%)\end{array}$ & $\begin{array}{c}270 \\
(58,3 \%)\end{array}$ & $\begin{array}{c}178 \\
(38.4 \%)\end{array}$ \\
\hline $\begin{array}{l}\text { 2. Los hombres homosexuales se estimulan } \\
\text { olfativamente por las hormonas masculinas y los } \\
\text { hombres heterosexuales por las hormonas } \\
\text { femeninas. }\end{array}$ & Correcta & $\begin{array}{c}65 \\
(14.0 \%)\end{array}$ & $\begin{array}{c}111 \\
(24.0 \%)\end{array}$ & $\begin{array}{c}287 \\
(62.0 \%)\end{array}$ \\
\hline $\begin{array}{l}\text { La homosexualidad y el lesbianismo } \\
\text { esentan las mismas diferencias cerebrales. }\end{array}$ & Incorrecta & $\begin{array}{c}28 \\
(6.0 \%)\end{array}$ & $\begin{array}{c}146 \\
(31.5 \%)\end{array}$ & $\begin{array}{c}289 \\
(62.4 \%)\end{array}$ \\
\hline $\begin{array}{l}\text { Los cerebros de hombres heterosexuales y } \\
\text { mosexuales son diferentes y los cerebros de } \\
\text { ujeres heterosexuales y lesbianas son más } \\
\text { recidos. }\end{array}$ & Correcta & $\begin{array}{c}11 \\
(2.4 \%)\end{array}$ & $\begin{array}{c}242 \\
(52.3 \%)\end{array}$ & $\begin{array}{c}210 \\
(45.4 \%)\end{array}$ \\
\hline $\begin{array}{l}\text { Existe más conocimiento cerebral sobre la } \\
\text { mosexualidad que el lesbianismo. }\end{array}$ & Correcta & $\begin{array}{c}56 \\
(9.9 \%)\end{array}$ & $\begin{array}{c}106 \\
(22.9 \%)\end{array}$ & $\begin{array}{c}311 \\
(67.2 \%)\end{array}$ \\
\hline $\begin{array}{l}\text { Frente a estímulos eróticos femeninos la } \\
\text { tividad cerebral es diferente en mujeres } \\
\text { terosexuales. lesbianas y bisexuales. }\end{array}$ & Correcta & $\begin{array}{c}73 \\
(15.8 \%)\end{array}$ & $\begin{array}{c}101 \\
(21.8 \%)\end{array}$ & $\begin{array}{c}289 \\
62.4 \%)\end{array}$ \\
\hline $\begin{array}{l}\text { Existe un "gen gay" que determina la } \\
\text { mosexualidad y el lesbianismo. }\end{array}$ & Incorrecta & $\begin{array}{c}10 \\
(2.2 \%)\end{array}$ & & $\begin{array}{c}149 \\
(32.2 \%)\end{array}$ \\
\hline $\begin{array}{l}\text { tación sexual estaría influenciada por } \\
\text { as sexuales durante el embarazo. }\end{array}$ & Correcta & & & $\begin{array}{c}219 \\
(47.3 \%)\end{array}$ \\
\hline $\begin{array}{l}\text { Existen más hombres homosexuales que } \\
\text { ujeres lesbianas. }\end{array}$ & Correcta & $\begin{array}{c}18 \\
(3.9 \%)\end{array}$ & $\begin{array}{c}64 \\
(13.8 \%)\end{array}$ & $\begin{array}{c}381 \\
(82.3 \%)\end{array}$ \\
\hline $\begin{array}{l}\text { 10. Cuando un hombre es homosexual existen } \\
\text { más probabilidad que su hermano también sea } \\
\text { homosexual. }\end{array}$ & Correcta & 7 & & $\begin{array}{c}133 \\
(28.7 \%)\end{array}$ \\
\hline $\begin{array}{l}\text { La predisposición genética de la } \\
\text { mosexualidad y el lesbianismo es la misma. }\end{array}$ & Inc & $\begin{array}{c}30 \\
(6.5 \%)\end{array}$ & & $\begin{array}{c}302 \\
(65.2 \%)\end{array}$ \\
\hline $\begin{array}{l}\text { Existen más mujeres lesbianas que mujeres } \\
\text { sexuales. }\end{array}$ & Ince & $\begin{array}{c}19 \\
(4.1 \%)\end{array}$ & $(16$ & $\begin{array}{c}368 \\
(79.5 \%)\end{array}$ \\
\hline $\begin{array}{l}\text { 3. Cuando una mujer es lesbiana existen más } \\
\text { obabilidad que su hermana también sea } \\
\text { sbiana }\end{array}$ & Correcta & $\begin{array}{c}6 \\
(1.3 \%\end{array}$ & & $\begin{array}{c}140 \\
(30.2 \%)\end{array}$ \\
\hline $\begin{array}{l}\text { La identidad de género depende de regiones } \\
\text { rebrales y como estas hacen que una persona } \\
\text { rriba su cuerpo. }\end{array}$ & Correcta & $\begin{array}{c}102 \\
(22.0 \%)\end{array}$ & $\begin{array}{c}78 \\
(16.8 \%)\end{array}$ & $\begin{array}{c}283 \\
(61.1 \%)\end{array}$ \\
\hline $\begin{array}{l}\text { Las personas transgéneros son homosexuales } \\
\text { bisexuales en igual porcentaje que las personas } \\
\text {--transgéneros. }\end{array}$ & Inco & $\begin{array}{c}24 \\
(5.2 \%)\end{array}$ & $\begin{array}{c}95 \\
(20.5 \%)\end{array}$ & $\begin{array}{c}344 \\
(74.3 \%)\end{array}$ \\
\hline $\begin{array}{l}\text { Las personas transgéneros poseen una } \\
\text { coherencia entre sus rasgos físicos y su imagen } \\
\text { rporal en las regiones sensitivas del cerebro. }\end{array}$ & Corre & $\begin{array}{c}83 \\
(17.9 \%)\end{array}$ & $\begin{array}{c}90 \\
(19.4 \%)\end{array}$ & $\begin{array}{c}290 \\
(62.6 \%)\end{array}$ \\
\hline $\begin{array}{l}\text { 7a región cerebral relacionada con el } \\
\text { mportamiento sexual es diferente en mujeres } \\
\text { terosexuales y mujeres transexuales } \\
\text { signados como hombres al nacer). }\end{array}$ & Inc & $\begin{array}{c}24 \\
(5.2 \%)\end{array}$ & $\begin{array}{c}92 \\
(19.9 \%)\end{array}$ & $\begin{array}{c}347 \\
(74.9 \%)\end{array}$ \\
\hline $\begin{array}{l}\text { 18. Las regiones cerebrales relacionadas con la } \\
\text { percepción del propio cuerpo son iguales en } \\
\text { personas transexuales y personas no- } \\
\text { transexuales. }\end{array}$ & Inc & $\begin{array}{c}63 \\
(13.6 \%)\end{array}$ & $\begin{array}{c}66 \\
(14.3 \%)\end{array}$ & $\begin{array}{c}334 \\
(72.1 \%)\end{array}$ \\
\hline
\end{tabular}

estudiantes solteros y lo que se encuentran en una relación, creencia religiosa, estudiantes con amigos/as o conocidos/as homosexuales o bisexuales de la muestra y estudiantes que no, estudiantes con amigos/as o conocidos/as transgéneros y estudiantes que no, estudiantes que recibieron y no recibieron educación sexual en su colegio o universidad, entre estudiantes que recibieron y no recibieron información sobre la homosexualidad e identidad de género recibida en clases de educación sexual e ingreso mensual.

En la tabla 4 se muestra el análisis comparativo entre los puntajes obtenidos en la escala EHF-10, la escala EANT y el cuestionario CBHT según la orientación sexual de la muestra. Es posible notar que en dos dimensiones y el puntaje total de la escala EHF-10 los/as estudiantes de educación física que se declaran heterosexuales poseen puntajes significativamente más altos que los homosexuales y bisexuales. La misma situación ocurre con el puntaje total de la escala EANT. En cuatro de las seis dimensiones y en el puntaje total del cuestionario CBHT los/as estudiantes que se decla- ran homosexuales o bisexuales poseen más porcentaje de respuestas correctas en comparación con aquellos heterosexuales.

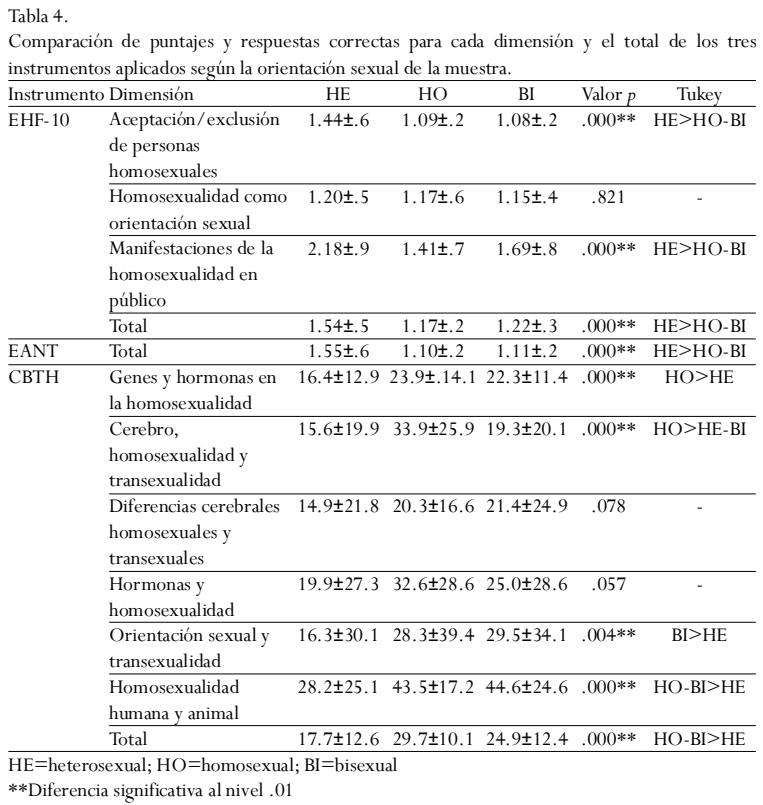

El porcentaje total de respuestas correctas del cuestionario $\mathrm{CBTH}$ no se correlaciona significativamente con el puntaje obtenido en la escala EHF-10 $\left(\mathrm{r}^{2}=-.003\right.$; $p=.131$ ), pero lo hace en forma negativa y baja, con los puntajes de la escala EANT $\left(\mathrm{r}^{2}=-.010 ; p=.018\right)$. En este último, el modelo de regresión lineal muestra una constante con un valor $\mathrm{B}=22.590$ con un error estándar $=1.542$ y el puntaje de la escala EANT con un valor $\mathrm{B}=-2.278$ y un error estándar $=.959$.

En la tabla 5 se observan los análisis de regresiones lineales jerárquicos que revela que la orientación sexual, el tener amigos/as o conocidos/as homosexuales o bisexuales, tener amigos/as o conocidos/as transgéneros y las creencias religiosas son predictores de los puntajes de la escala EHF-10; la orientación sexual, el tener

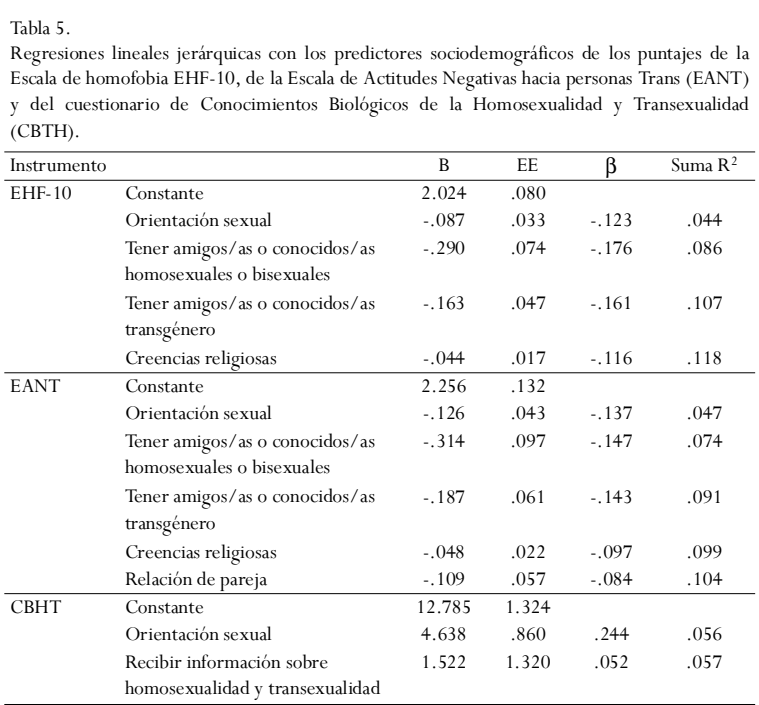


amigos/as o conocidos/as homosexuales o bisexuales, tener amigos/as o conocidos/as transgéneros, las creencias religiosas y el estado estar en una relación de pareja son predictores de los puntajes de la escala EANT; la orientación sexual y el recibir información sobre la homosexualidad y transexualidad en clases de educación sexual son predictores de los puntajes en el cuestionario CBHT.

\section{Discusión}

Los resultados revelan que los hombres presentan niveles más altos de homofobia frente a situaciones como el miedo a sentirse atraído por alguien del mismo sexo o la exhibición en público de orientaciones no heterosexuales. Trabajos como el de Guevara (2020) y Silva et al. (2018) explican que es posible presentar bajos niveles de homofobia cuando se presenta como algo ajeno a nosotros, pero mostrar rechazo o discriminación cuando el sujeto se ve envuelto. El hecho que los hombres presenten mayores niveles de rechazo frente a la situación de sentirse atraído por alguien del mismo sexo es algo habitual, producto de la influencia sociocultural, donde los signos de feminización en los hombres se consideran como debilidad y fracaso (Nieto-Gutiérrez et al., 2019), situación que se acentúa en el deporte y la educación física (O’Brien et al., 2013).

Otras variables como el año cursado de la carrera de Pedagogía en Educación Física, el nivel socioeconómico o tener pareja no poseen incidencias sobre los niveles de homofobia reportados por la muestra. Al contrario de lo esperado, la educación sexual parece ser contraproducente, ya que aquellos que dijeron recibirla son los que poseen índices más elevados de homofobia. En el presente estudio se observó que un bajo porcentaje de estudiantes que recibió educación sexual, recibió información sobre homosexualidad y transexualidad, ya que este tipo de educación recibida en colegios se suele restringir a prevención de embarazo y enfermedades de transmisión sexual (González y Riquelme, 2018), generalmente con una visión conservadora, lo cual podría influir en que aquellos que dicen haber recibido educación sexual presenten mayores niveles de rechazo hacia las diversas orientaciones sexuales.

En el presente estudio los cuatro factores más influyentes sobre la homofobia son la orientación sexual, la convivencia con amigos/as o conocidos/as homosexuales o bisexuales, la convivencia con amigos/as o conocidos/as transgénero y las creencias religiosas, ya que aquellos que reportan ser homosexuales o bisexuales, conocer personas con distintas orientaciones sexuales e identidad de género y los que dicen ser ateos/as presentan los índices más bajos de rechazo a homosexuales. Estos resultados son similares a los reportados en otros estudios (Colonnello et al., 2020, Georgius et al., 2018), donde factores conservadores como la religión tienden a rechazar conductas homosexuales, impidiendo la relación respetuosa con estas personas (McDermott et al., 2014), y factores como la convivencia con personas homosexuales o bisexuales, que representan factores protectores, que ayudan a la tolerancia y a un vivir más considerado con los otros.

En relación con los resultados de los niveles de transfobia de la muestra resultan ser similares a los de homofobia, sin incidencia del curso, edad, nivel socioeconómico o educación sexual recibida. Nuevamente la orientación sexual, convivencia con amigos/as o conocidos/as transgéneros y las creencias religiosas son los factores más influyentes, presentando menores niveles de transfobia los/as estudiantes que se reportan como ateos/as y los/as que conocen personas trans.

Los factores más influyentes sobre los conocimientos biológicos de la homosexualidad y transexualidad son la orientación sexual y recibir información sobre estos temas en las clases de educación sexual. Los/as estudiantes que declararon ser homosexuales o bisexuales mostraron tener mayor conocimiento sobre la biología de estas conductas, lo cual podría explicarse debido a una mayor búsqueda de información e interés en el tema debido a las experiencias que vivencian. Sin embargo, estas variables solo pueden explicar el 6\% de los resultados obtenidos.

Frente a los conocimientos biológicos sobre la homosexualidad y transexualidad, la muestra revela bajos niveles de instrucción con sólo dos preguntas que superan el 30\% de respuestas correctas y con 6 ítems que no alcanzan el 10\% de aciertos. Ninguna de las variables estudiadas presenta incidencia sobre los niveles de conocimientos estudiados, lo cual podría explicarse por la poca difusión y nula entrega de antecedentes biológicos sobre la orientación sexual e identidad de género que ocurre en clases de educación sexual en los diversos programas educativos (González et al., 2015). Esto se refleja en que existe una independencia entre aspectos socioculturales y conocimientos sobre las bases biológicas de la homosexualidad y transexualidad. Lo anterior también explicaría la baja relación entre dichos conocimientos y los niveles de homofobia y transfobia en la muestra, pese a que autores como Piskur y Degelman 
(1992) y Fernández (2009) argumentan que mayores conocimientos sobre la naturaleza de la orientación sexual repercuten en una disminución de la homofobia.

Finalmente, es necesario revisar la implementación de la educación sexual en los establecimientos educacionales, pues según la Ley $\mathrm{N}^{\circ} 20.418$ de Chile en el artículo primero obliga a todos los colegios que reciben subvención del estado a contar con programas de educación sexual. En este sentido, existen objetivos relacionados con la presente investigación que orientan al diseño e implementación como conocer y respetar los diferentes tipos de orientaciones sexuales e identidad de género y también entender que el desarrollo de la sexualidad puede ser diferente entre las personas (MINEDUC, 2017), no obstante, se puede apreciar desde los resultados de este estudio, que futuros profesionales de la educación no están preparados en dichas temáticas para posteriormente educar al alumnado.

\section{Conclusión}

La muestra evaluada presenta bajos niveles de homofobia y transfobia, siendo las variables orientación sexual, creencias religiosas y convivencia con conocidos/as o amigos/as homosexuales, bisexuales o transexuales las que presentan mayor incidencia sobre el rechazo hacia personas de diversas orientaciones sexuales e identidad de género. Las personas que se reconocen como ateos/as y las que conviven con dichas personas presentan los niveles más bajos de homofobia y transfobia. Con respecto a los conocimientos biológicos sobre la homosexualidad y transexualidad, la muestra presenta valores muy bajos, con pocos ítems con más de un 20\% de aciertos. Lo anterior repercute en una relación baja y negativa entre dichos conocimientos y las actitudes negativas hacia personas homosexuales y trans.

Son necesarias investigaciones en muestras de otras carreras de pedagogía para tener una mirada más amplia de las actitudes negativas hacia la homosexualidad y personas trans por parte de los futuros profesores en todo Chile.

\section{Referencias}

Acker, G. (2017).Transphobia among students majoring in the helping professions. Journal of Homosexuality, 64(14), 2011-2029. https://doi.org/10.1080/ 00918369.2017.1293404

Álvarez-Conde, T., y Rodríguez-Castro, Y. (2017). Eva- luación de los conocimientos y actitudes hacia la sexualidad de futuros/as docentes de educación primaria. Revista de Estudios e Investigación en Psicología y Educación, 6, 1-5. https://doi.org/10.17979/ reipe.2017.0.06.2332

American Psychological Association (2011). Definition of terms: Sex, Gender, Gender Identity, Sexual Orientation. Disponible en: http://www.apa.org/pi/lgbt/ resources/sexualitydefinitions.pdf

Barrientos, J., Espinoza-Tapia, R., Meza, P., Saiz, J., Cárdenas, M., Guzmán-González, M., Gómez, F., Bahamondes, J. \& Lovera, L. (2019). Efectos del prejuicio sexual en la salud mental de personas transgénero chilenas desde el modelo de estrés de las minorías: una aproximación cualitativa. Terapia Psicológica, 37(3), 181-197.

Campo, A., Herazo, E. \& Oviedo, H. (2013). Sustantivos para definir homofobia. Revista Ciencias de la Salud, 11(3), 297-294.

Colonnello, E., Toccini, L., Ciocca, G., Limoncin, E., Moscato, G., Andreoni, M. \& Jannini, E. (2020). Attitudes and knowledge towards homosexuality: an observational study on a sample of medical students. Journal of Sexual Medicine, 17(6), S123. https://doi.org/10.1016/j.jsxm.2020.04.019

Fernández, A. (2009). Bases biológicas de la preferencia sexual. Ciencia, 60(2), 23-36.

García-Acosta, J., Castro-Peraza, M., Perestelo-Pérez, L., Rivero-Santana, A., Arias-Rodríguez, Á., Lorenzo-Rocha, N. (2020). Midiendo las actitudes negativas y la transfobia explícita en un servicio público de salud. Index de Enfermería, 29(4), e12765.

García-Barba, M., Serrano-Moragón, N., Camañes Martorell, J., Ruiz-Palomino, E., \& Ballester-Arnal, R. (2018). Actitudes hacia la transexualidad en jóvenes. Ágora de Salut, 5, 11-19. http://dx.doi.org/ 10.6035/AgoraSalut.2018.5.1

Georgius, Y., Patsantaras, N. \& Kamberidou, I. (2018). Homophobia predictors - a case study in Greece: heterosexual physical education student attitudes towards male and female homosexuality. Journal of Physical Education and Sport, 18(S2), 1209-1216. https: / /doi.org/10.7752/jpes.2018.s2180

González, E., Molina, T. \& Luttges, D. (2015). Características de la educación sexual escolar recibida y su asociación con la edad de inicio sexual y uso de anticonceptivos en adolescentes chilenas sexualmente activas. Revista Chilena de Obstetricia y Ginecología, 80(1), 24-32. http://dx.doi.org/10.4067/S071775262015000100004 
González, E. \& Riquelme, R. (2018). Características de los Programas de Educación Sexual en Liceos de la provincia de Santiago, Chile. Cuadernos Médico Sociales, 58(2), 23-30.

Guevara, R. (2020). Los roles de género y las actitudes frente a la homosexualidad masculina en estudiantes universitarios heterosexuales del primer y séptimo semestre de la carrera de Pedagogía de la Actividad Física y Deportes, de la Facultad de Ciencias Humanas y de la Educación, de la Universidad Técnica de Ambato, Cantón Ambato, provincia de Tungurahua. Seminario para optar al título de Psicólogo Educativo y Orientador Vocacional, Universidad Técnica de Ambato, Ecuador.

Hatibovic, F., Bobowik, M., Faúndez, X. \& Sandoval, J. (2017). Xenofobia y homofobia como efectos de la orientación política, religión y sexo mediados por clasismo y patriocentrismo en jóvenes universitarios chilenos. Revista Colombiana de Psicología, 26(1), $131-148$. https: / / doi.org/10.15446/ rcp.v26n1.55687

Kwak, H., Kim, M. \& Kim, M. (2019). Severity and influencing factors of homophobia in korean nursing students. Int.J. Environ. Res. Public Health, 16(23), 4692. https://doi.org/10.3390/ijerph16234692

Maureira, F. (2021). Conocimientos biológicos de la homosexualidad y transexualidad en estudiantes de Educación Física de Chile. Retos, 42, 805-812. https: / /doi.org/10.47197/retos.v42i0.88708

Maureira, F. (2020). Amor, sexo y cerebro. Bubok Publishing.

Maureira, F., Escobar, N., Flores, E., Bahamondes, V., Hadweh, M. \& Serey, D. (2022). Actitudes hacia la homosexualidad y transexualidad en estudiantes de Educación Física de Chile. Retos, 43, 46-52. https: / / doi.org/10.47197/retos.v43i0.88969

Maureira, F. \& Flores, E. (2018). Manual de investigación cuantitativa. Bubok Publishing.

McDermott, R., Schwartz, J., Lindley, L. \& Proietti, J. (2014). Exploring men's homophobia: Associations with religious fundamentalism and gender role conflict domains. Psychol Men Mascul, 15(2), 191-200. https: / / doi.org/10.1037/a0032788

MINEDUC (2017). Educación en sexualidad, afectividad y género. Disponible en: https:// convivenciaescolar.mineduc.cl/wp-content/ uploads /2019/04/Educaci\%C3\%B3n-en-Sexualidad-Afectividad-y-G\%C3\%A9nero.-Mineduc2017.pdf

Moral, J. \& Ortega, M. (2008). Diferencias de género en representación social de la sexualidad, así como en actitudes y conductas sexuales. Estudios sobre las
Culturas Contemporáneas, 14(28), 97-119.

Nieto-Gutiérrez, W., Komori-Pariona, J., Sánchez, A., Centeno-Leguía, D., Arestegui-Sánchez, L., De la Torre-Rojas, K., Niño-García, R., MendozaAucaruri, L., Mejia, C. \& Quiñones-Laveriano, D. (2019). Factores asociados a la homofobia en estudiantes de medicina de once universidades peruanas. Rev Colomb Psiquiat, 48(4), 208-214. https: / / doi.org/ 10.1016/j.rcp.2018.01.003

Oltra, J., Huluta, E., Rodríguez-Fernández, A. \& GarcíaCueto, E. (2017). Desarrollo de un test de homofobia. Revista Electrónica de Metodología Aplicada, 22(2),1629.

O’Brien, K., Shovelton, H. \& Latner, J. (2013). Homophobia in physical education and sport: The role of physical/sporting identity and attributes, authoritarian aggression, and social dominance orientation. International Journal of Psychology, 48(5), $891-899$. https: / / doi.org/10.1080/ 00207594.2012.713107

Páez, J., Hevia, G., Pesci, F. \& Rabbia, H. (2015). Construcción y validación de una escala de actitudes negativas hacia personas trans. Revista de Psicología (PUCP), 33(1), 153-190.

Piskur, J. \& Degelman, D. (1992). Effect of reading a summary of research about biological basis of homosexual orientation on attitudes toward homosexuals. Psychological Reports, 71, 1219-1225. https: / /doi.org/10.2466/pr0.1992.71.3f.1219

Silva, P., Jaeger, A. \& Valdivia, P. (2018). Percepción de los estudiantes sobre comportamientos homofóbicos y heterosexistas en educación física. Revista de Psicología del Deporte, 27(2), 39-46.

World Medical Asociation. (2013). World Medical Association Declaration of Helsinki Ethical Principles for Medical Research Involving Human Subjects. JAMA, 310(20), 2191-2194. https://doi.org/ 10.1001/jama.2013.281053

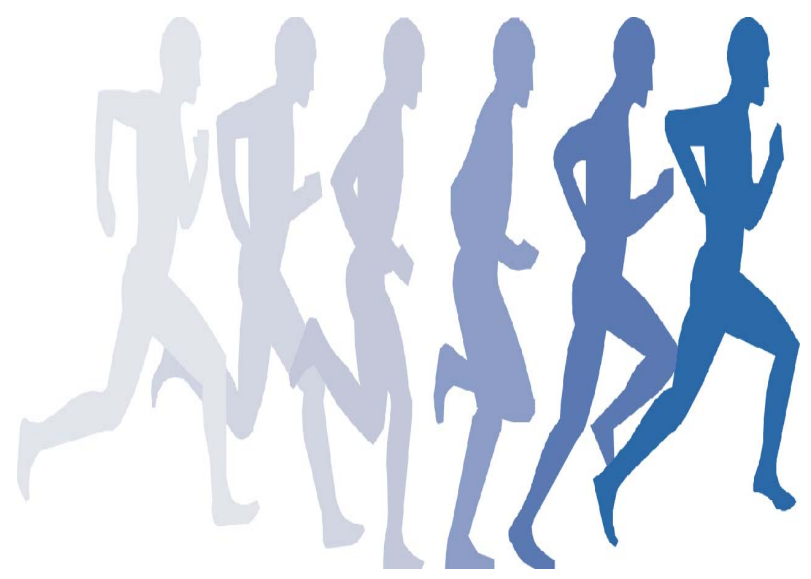

